\title{
A New Frequency Dependent Approach to
} Model Validation for Iterative Identification

\section{and Control Schemes}

\author{
P. Balaguer and R. Vilanova
}

\begin{abstract}
Classical validation methods "accept" or "reject" a model as a valid representation of a plant for some intended use. However this binary result has several problems as firstly, models are neither good nor bad but have a certain valid frequency range and secondly the procedure gives no insight into why the model is not useful or how to improve the model. Moreover within the framework of iterative identification and control design the model validation issue arises the following requirements: i) Is it possible to improve an existing model?. ii) How can the model be improved?. iii) How authoritative can be the designed controller?. These facts question the suitability of traditional model validation schemes in general and their suitability for iterative control schemes in particular. We present a new validation procedure that overtakes these problems by performing the model validation frequency dependent. The validation procedure is then more informative due to its frequency information content. As a result the same model can be validated for some frequency band and invalidated for a distinct frequency range.
\end{abstract}

\section{Index Terms}

Model Validation, Frequency Domain, Iterative Control, Control Oriented

\section{INTRODUCTION}

In order to have confidence in a model, it is necessary to validate it. Different model validation approaches exist. Their difference is based upon the assumptions about the plant and

The authors are with the Telecommunication and System Engineering Department, ETSE, Universitat Autònoma de Barcelona, 08193 Bellaterra, Barcelona, Spain.

Corresponding author pedro.balaguer@uab.es 
models. Classical validation methods, based on classical model identification ([1] and [2]) rely on statistical uncertainty assumptions due to stochastic noise only. On the other hand, control oriented identification methods [3] (i.e. $H_{\infty}$ identification, stochastic embedding, set membership identification, etc.) lead to validation assumptions based on bounded noise and bounded model undermodelling. However in both cases the output of the validation process is just a "validated/invalidated" result.

Regarding classical validation methods, the problem of assessing the validity of an identified model has been traditionally linked with the problem of model order selection. The classical model validation literature ([1] and [2]) has approached the problem in two ways:

- Use of plots and common sense.

- Use of statistical tests on the residuals (i.e. the difference between the real output and the model output).

The first approach is basically based on the comparison of experimental data with the model output. If both are similar then the model can be considered a good one. However there are two unavoidable reasons that prevent the model output to fit data perfectly: the modelling errors and perturbations.

The second approach is to apply a hypothesis test over the residual. A hypothesis test is a statement about a random variable. This statement is expressed by means of two hypothesis $H_{0}$ and $H_{1} . H_{0}$ is called the null hypothesis and $H_{1}$ is the alternative hypothesis. In order to decide the validity of either the null hypothesis $H_{0}$ or the alternative hypothesis $H_{1}$, an estimation of a population parameter (e.g. mean or variance) is computed from a population sample and it is compared against the assumed population parameters. These population parameters are random variables too with certain mean and variance. If it is likely that the computed statistic is inside the population parameters distribution then $H_{0}$ is accepted, otherwise $H_{0}$ is rejected in favour of $H_{1}$. As a result, two errors are possible: to reject $H_{0}$ when it is true, that is a false alarm (type I error or $\alpha$ error), or to fail to reject $H_{0}$ when $H_{0}$ is false (type II error or $\beta$ error).

The null hypothesis (i.e. $H_{0}$ ) taken on the model validation test is the hypothesis on the residual $\xi(t)$, which follows from the assumptions on the disturbance. The more common assumptions over the residual are [2]:

h1: $\xi(t)$ is a zero mean white noise.

h2: $\xi(t)$ has a symmetric distribution. 
h3: $\xi(t)$ is independent of past inputs $E \xi(t) u(\tau)=0, t>\tau$.

h4: $\xi(t)$ is independent of all inputs $E \xi(t) u(\tau)=0, \forall t, \tau$.

The above assumptions lead to check two main properties, the whiteness of the residuals (i.e. $\mathrm{h} 1, \mathrm{~h} 2)$ and the cross-correlation between residuals $\xi(t)$ and control actions $u(t)$ (i.e. h3, h4).

Hence classical validation tests can be classified as follows

- Whiteness Test.

- Autocorrelation test. (h1)

- Testing changes of sign. (h1 and h2)

- Independence between residuals and inputs.

- Cross-correlation test of past inputs. (h3) or (h3 and h1)

- Cross-correlation test of all inputs. (h4) or (h4 and h1)

The rationale of the tests is to detect causes of variation on the residual distinct than the ones assumed. For example if the residual is assumed to be white noise and the test shows that the whiteness statistical hypothesis is violated then we assume that there is a distinct cause causing the mismatch (i.e. model error).

The result of the statistical tests above reviewed is a binary one. In fact the test either validates or invalidates the model. No further information is provided by the test. As a result, two important drawbacks are:

1) There is no information on important model aspects such as [4]:

- The reasons why the model is invalidate.

- How to improve the model.

- The model usefulness degree.

2) In iterative identification and control schemes undermodelling is normally present [5]. In fact as stated in [6]: "For such a model (a model simpler that the one that minimizes the total error) typically the bias error is the dominating contribution to the total error. Consequently, such models would normally be falsified during model validation. These are then reduced complexity models".

Thus, as a conclusion, although the theory of classical validation methods is well developed and plenty of successful applications, it has limitations when a more informative validation procedure is required, as for example in iterative identification and control approaches. 
Iterative identification and control design schemes improve performance by designing new controllers on the basis of new identified models [7]. The procedure is as follows: an experiment is performed in closed loop with the current designed controller. A new model is identified with the experimental data and a new controller is designed using the new model. The procedure is repeated until satisfactory performance is achieved.

The rationale behind iterative control is that if iteratively "better" models are identified, hence "better" performing controllers can be designed. However the meaning of "better" model needs some clarification. The idea of modelling the "true" plant has proven to be bogus [8]. Instead a good model for control is one that captures accurately the interesting frequency range for control purposes. In fact the model has no other use than to design a controller, thus the use of the model is instrumental [9]. Hence, once a model is obtained it is necessary to validate it. On the iterative identification and control schemes this should be done each time a new model is identified (i.e. at each iteration).

The main problem of the validation methods reviewed is that the answer is a binary result (i.e. validated/invalidated). However models are neither good nor bad but have a certain valid frequency range (e.g. normally models are good at capturing low frequency behavior but their accuracy degrades at higher frequencies). Moreover the iterative identification and control procedures have their own particular requirements:

- Is it possible to improve an existing model?. Is the data informative enough to attempt a new identification?.

- How can the model be improved?. Is the model order/structure rich enough to capture the interesting features of the plant?.

- How authoritative can be the controller designed on the basis of the new model?. Which is the validity frequency range of my model?.

The above requirements for iterative control can not be provided by the classical model validation approaches above introduced because:

- No indication on the possibility to improve an existing model. This problem is solved in [9] by the use of classical validation methods (i.e. crosscorrelation test) together with the visual comparison of two power spectrum.

- In iterative identification and control approaches a low order model is fitted to capture 
the frequency range of interest for control. Hence undermodelling is always present. This fact makes it difficult to apply traditional model validation schemes as the output of the validation procedure is a binary answer (i.e. validated/no validated) [6].

- No indication on how to improve the model on the next iteration (i.e. model order selection and/or input experiment design).

- No indication on the model validity range for control design (i.e. controller bandwidth selection).

Summing up, these arguments question the suitability of classical validation approaches in general and its application to iterative identification and control schemes in particular.

In this article we propose a new model validation algorithm in order to solve the above mentioned problems. The validation result is no longer"validated/invalidated" but frequency dependent. Thus the validation result is more informative as the model can be validated for some frequency range and the same model can be invalidated for a distinct frequency band.

The article contributions are organized as follows:

- A new procedure for model validation in the frequency domain is presented (Section IIB). This procedure permits to validate or invalidate models over certain frequency ranges. The procedure is the translation of a time domain residual whiteness test to a frequency dependent residual whiteness test. The counterpart on the frequency domain of a time domain whiteness test is established (Section II-A). This leads to stating the statistical properties of each spectrum frequency component if the time domain transformed signal is a white noise. It is shown that the normalized spectrum is a random variable with a $\chi^{2}$ distribution of 2 degrees of freedom (Theorem 2). The validation/invalidation step is based on a hypothesis test applied to each frequency component. This determines if certain frequency components have an unusual content that discards the model validity for this frequency value. The acceptance/rejection decision of the frequency component validity comes with a probability measure (Section II-C).

- The intended use of the model (e.g. open loop or closed loop) is taken into account by considering the structure from which the residuals are generated. In Section III a study of the residuals information content and its statistical properties for different structures (e.g. open loop Vs closed loop) is conducted. As the validation procedure is based on a whiteness test, the residual should be white noise whenever the model fits accurately the plant. This 
however is not the general case when residuals coming from closed loop comparisons are used. In order to overcome this difficulty, a novel structure is proposed in Section III-B which is able to provide white residuals when two closed loops are compared.

- The benefits of the presented validation procedure on iterative identification and control schemes is discussed in Section IV. It is shown that the validation procedure shifts from a classical static test (i.e. validation/invalidation) to a dynamic one which gives frequency domain information useful for improvement of identification and control design on iterative schemes.

- The usefulness of the presented procedure is presented by means of three examples (Section V). The first example presents the application to an open loop model validation. It is shown how the reliable model bandwidth is calculated with our algorithm. The second and third example deal with iterative control approaches, and how our algorithm is helpful to decide the appropriate bandwidth of the controller to be designed and the input design for future identification experiments.

\section{FREQUENCY DEPENDENT MODEL VALIDATION}

The main objective of the paper we present is to validate a model on the frequency domain. To this end a time domain validation procedure based on testing the residual whiteness is modified to achieve the pursued objectives. The idea is as follows. It is assumed that if the residual is white noise the model is validated because the residual contains no further useful information that could be used to improve the model accuracy. This test is usually performed in the time domain by studying the residual autocorrelation, the number of sign changes, etc [1].

We translate the time domain residual to the frequency domain by its discrete Fourier transform. Moreover, the statistical properties of the spectrum of a white noise signal are calculated. The objective is to test if the spectrum calculated from the residual has properties of a white noise. As a result, one unique test in the time domain has been translated to $N$ different tests in the frequency domain. We check if the $k^{\text {th }}$ frequency component of the spectrum has the properties of a typical frequency component of a white noise. In case of an affirmative answer, we have no reason to believe that the model is invalidated on that frequency component. On the other hand, if there are certain frequency components which clearly do not behave accordingly to the statistical properties of white noise then it is likely that at this frequency range there is 
an important mismatch between the model and the plant. As a result the model is invalidated for that frequency range.

\section{A. Whiteness Test on the Frequency Domain}

In this section the statistical time domain properties of a white noise are translated to the frequency domain. This is accomplished by means of two theorems. The first one is an intermediate result that is used by the second theorem which describes the frequency domain distribution of the spectrum of a white noise.

Theorem 1: Let $\xi(n)$ be a sequence of independent identically distributed (IID) samples of normal distribution $N\left(\mu_{\xi}, \sigma_{\xi}^{2}\right)$. If we express the discrete Fourier transform by its real and imaginary part, that is $\xi_{k}=R_{k}+j I_{k}=\frac{1}{N} \sum_{n=0}^{N-1} \xi(n) e^{-j \Omega_{0} k n}$, then the real part $R_{k}$ is a random variable normally distributed $\left(R_{k} \in N\left(\mu_{R_{k}}, \sigma_{R_{k}}^{2}\right)\right.$ ) with mean $\mu_{R_{k}}$ and variance $\sigma_{R_{k}}^{2}$ given by

$$
\begin{aligned}
\mu_{R_{k}} & =\mu_{\xi} \frac{1}{N} \sum_{n=0}^{N-1} \cos \left(\Omega_{0} k n\right) \\
\sigma_{R_{k}}^{2} & =\sigma_{\xi}^{2} \frac{1}{N^{2}} \sum_{n=0}^{N-1} \cos ^{2}\left(\Omega_{0} k n\right)
\end{aligned}
$$

Similarly the Imaginary part $I_{k}$ is a random variable normally distributed $I_{k} \in N\left(\mu_{I_{k}}, \sigma_{I_{k}}^{2}\right)$ with mean $\mu_{I_{k}}$ and variance $\sigma_{I_{k}}^{2}$ given by

Proof: See Appendix VII-A

$$
\begin{aligned}
\mu_{I_{k}} & =\mu_{\xi} \frac{1}{N} \sum_{n=0}^{N-1} \sin \left(\Omega_{0} k n\right) \\
\sigma_{I_{k}}^{2} & =\sigma_{\xi}^{2} \frac{1}{N^{2}} \sum_{n=0}^{N-1} \sin ^{2}\left(\Omega_{0} k n\right)
\end{aligned}
$$

Remark 1: $\mu_{R_{k}}$ is equal to zero for $k \in\{1,2, \ldots, N-1\}$ and $\mu_{R_{0}}$ equals the mean value of the residual (i.e. $\mu_{R_{0}}=\mu_{\xi}$ ). $\mu_{I_{k}}$ is always equal to zero for $k \in\{0,1,2, \ldots, N-1\}$

Theorem 2: The normalized squared gain $M_{k}^{2}$ defined as

$$
M_{k}^{2}=\left(\frac{R_{k}-\mu_{R_{k}}}{\sigma_{R_{k}}}\right)^{2}+\left(\frac{I_{k}-\mu_{I_{k}}}{\sigma_{I_{k}}}\right)^{2}
$$

has a $\chi^{2}$ distribution of 2 degrees of freedom if $R_{k}$ and $I_{k}$ are independent.

Proof: See Appendix VII-B 


\section{B. Procedure}

The frequency domain model invalidation procedure is as follows

1) Calculate the residual as the difference of the real output and the model estimated output $(\xi(n)=y(n)-\hat{y}(n))$.

2) Calculate the discrete Fourier transform of the residual $\left(\xi_{k}\right)$

3) Decompose each frequency component on its real part and imaginary part $\left(\xi_{k}=R_{k}+j I_{k}\right)$.

4) Calculate the distribution parameters of the Real and Imaginary part of the residual spectrum (i.e. $\mu_{R_{k}}, \mu_{I_{k}}, \sigma_{R_{k}}, \sigma_{I_{k}}$ ).

5) Calculate the normalized magnitude spectrum as follows

$$
M_{k}^{2}=\left(\frac{R_{k}-\mu_{R_{k}}}{\sigma_{R_{k}}}\right)^{2}+\left(\frac{I_{k}-\mu_{I_{k}}}{\sigma_{I_{k}}}\right)^{2}
$$

6) Perform an hypothesis test over the normalized magnitude spectrum calculated.
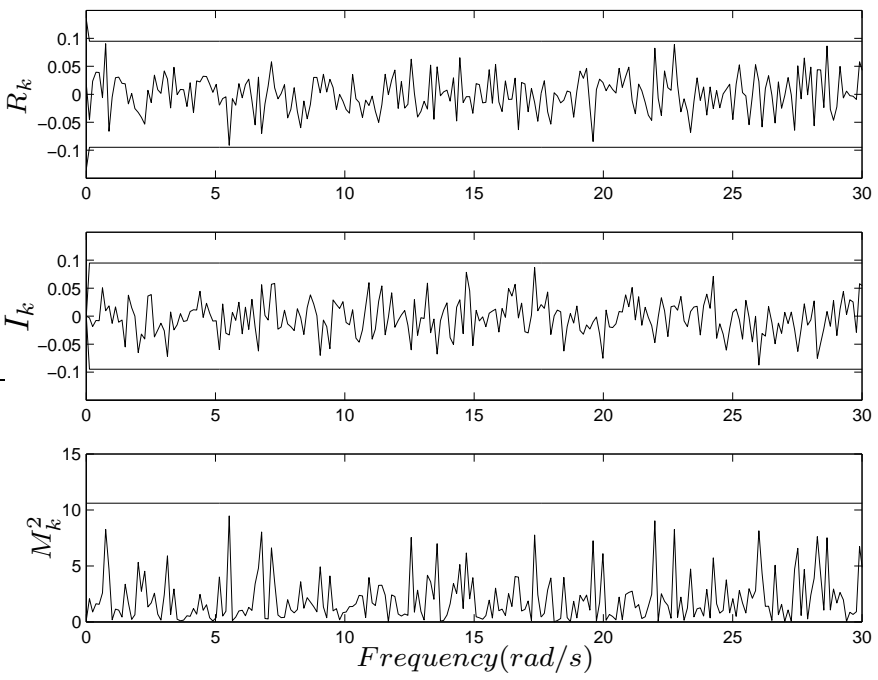

Fig. 1. White noise example

The above steps are materialized in the following example. A realization of a normally distributed random variable of zero mean and unity variance is performed with 500 samples. The discrete Fourier transform of the realization is calculated and decomposed into its real and imaginary parts (i.e. $R_{k}, I_{k}$ ). The values of $R_{k}$ and $I_{k}$ are shown in figure 1 . They follow a normal variable distribution with parameters given by equations (1)-(2) (i.e. $R_{k} \in N\left(\mu_{R_{k}}=\right.$ 
$\left.0, \sigma_{R_{k}}^{2}=0.001\right)$ and $I_{k} \in N\left(\mu_{I_{k}}=0, \sigma_{I_{k}}^{2}=0.001\right)$ ). On figure 1 the realizations of $R_{k}$ and $I_{k}$ are plotted together with the 3 sigma limits of their distribution (i.e. the $99.73 \%$ of the samples fall between the plotted limits). It can be seen that all the points fall inside this range.

Finally the normalized magnitude spectrum is calculated following equation (4). The magnitude spectrum can be seen in figure 1 together with the $99.5 \%$ confidence limit of the $\chi_{2}^{2}$ distribution (i.e. the $99.5 \%$ of the samples fall between 0 and 10.6). All the magnitude frequency components remain below of the confidence limit so there are no reasons to invalidate the model. The whiteness test has passed. In the sequent section the hypothesis test features are described more thoroughly.

\section{Hypothesis Test}

The hypothesis test is the last step of the presented procedure, where the decision of validation/invalidation of certain frequency component is taken. An hypothesis test is a statement expressed by means of two hypothesis $H_{0}$ and $H_{1} . H_{0}$ is called the null hypothesis and $H_{1}$ is the alternative hypothesis. The hypothesis test to be applied in our procedure is:

$$
\begin{aligned}
& H_{0}: M_{k}^{2} \in \chi_{2}^{2} \\
& H_{1}: M_{k}^{2} \notin \chi_{2}^{2}
\end{aligned}
$$

The hypothesis $H_{0}$ states that the normalized modulus $M_{k}^{2}$ of the $k^{t h}$ frequency component is $\chi_{2}^{2}$ distributed. On the other hand the hypothesis $H_{1}$ states that the normalized modulus $M_{k}^{2}$ of the $k^{\text {th }}$ frequency component is not $\chi_{2}^{2}$ distributed.

Remark 2: The hypothesis test stated in (5) is applied to each frequency component, from 0 $\mathrm{rad} / \mathrm{sec}$ up to the Nyquist frequency (i.e. $\pi / T s$, where $T s$ is the sample time).

In order to decide the validity of either the null hypothesis $H_{0}$ or the alternative hypothesis $H_{1}, M_{k}^{2}$ is computed. If it is "likely" that the value of $M_{k}^{2}$ lies inside the $\chi_{2}^{2}$ distribution then $H_{0}$ is accepted, otherwise $H_{0}$ is rejected in favour of $H_{1}$. As a result, two errors are possible: to reject $H_{0}$ when it is true, that is a false alarm (type I error or $\alpha$ error), or to fail to reject $H_{0}$ when $H_{0}$ is false (type II error or $\beta$ error).

The term "likely" introduced above is defined by the user by choosing the confidence limit. For example, if the confidence limit is chosen to be 10.6 then the $99.5 \%$ of the samples fall inside the limits. This confidence limit sets the type I error. Following the example presented 
in section II-B the type I error was of $0.5 \%$, that is the $0.5 \%$ of the samples of a $\chi_{2}^{2}$ must be greater than 10.6. The type II error is more difficult to be calculated as it depends on knowledge of of the actual distribution followed by $M_{k}^{2}$.

The hypothesis test is then simply a check that any magnitude of the normalized spectrum is less than the test limit. If the value is greater then it is very unlikely and the model results invalidate for this frequency.

\section{Control Oriented VAlidation}

Model validation theory is aimed towards checking the model usefulness for some intended use. Thus the model validation procedure should take into account the model use, for example control design or prediction purposes. In fact, it is recognized in [10] that arbitrary small model errors in open loop can lead to bad closed loop performance. On the other hand large open loop modelling errors do not necessarily lead to bad closed loop performance. As a result the model accuracy should be checked in such a way that the intended model use is taken into account in the model validation procedure.

An important aspect in the validation procedure to take into account the intended model use are the validation conditions. In fact validation from open loop data can provide a different result than validation with closed loop data. Furthermore it is completely different to validate an open loop model than to compare two closed loops, the one with the model and the real one (See for example [11]). This result points out the importance of the information that is being validated. This is accomplished by means of setting the experimental conditions from which data are generated.

In order to consider the model intended use in the validation procedure the conditions for data generation must be considered. In the following subsections different structures are proposed in order to compute the residuals and it is shown that they have considerable importance on the actual information that is validated. Its statistical properties are reviewed as the residuals must be statistically white under perfect model matching in order to apply the proposed algorithm. It is shown that the new model validation procedure introduced in this article can be endowed with the control oriented property. 


\section{A. Open Loop Validation (Stable Plants)}

The structure used to validate the model is shown in figure 6 .

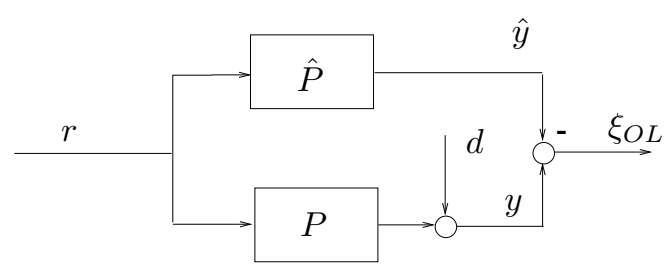

Fig. 2. Open Loop Model Validation

The residual is given by the following expression

$$
\xi_{O L}=d+(P-\hat{P}) r
$$

The residual $\xi_{O L}$ given by equation 11 is just the noise $d$ if the model and the plant are equal (i.e. $\hat{P}=P$ ). Hence the residual has the same stochastic properties than the noise. On the other hand if there exists a discrepancy between the model and the plant, a new term $((P-\hat{P}) r)$ appears in the residual. It should be remarked however that the model-plant error which will be detected is deeply dependent on the reference signal.

\section{B. Closed Loop Validation (Stable Plants)}

The proposed structure to validate stable models in closed loop is shown in figure 9 .

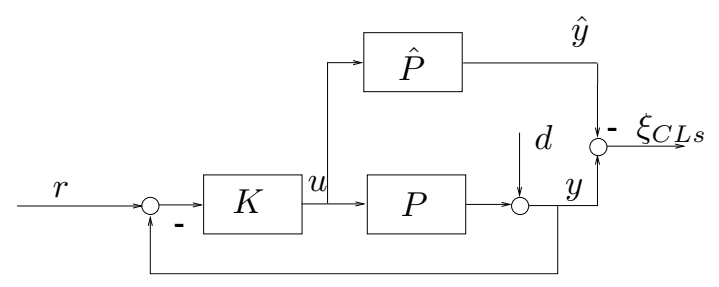

Fig. 3. Closed Loop Model Validation for Stable Plants

The residual is

$$
\xi_{C L s}=\frac{S}{\hat{S}} d+K S(P-\hat{P}) r
$$


If the model and the plant are equal (i.e. $\hat{P}=P$ ) then the two sensitivity functions ( $S=$ $(1+G K)^{-1}$ and $\left.\hat{S}=(1+\hat{G} K)^{-1}\right)$ are equal so the first term of equation 12 yields the noise $d$. Moreover the second term, under the same perfect model-plant matching assumption, is zero. Hence in this case the residuals are again the noise $d$.

If there exist a discrepancy between the model and the plant then the division between $S$ and $\hat{S}$ is no longer unity but a transfer function resulting from the noise $d$ filtered by $S / \hat{S}$ (i.e. autocorrelated). Additionally the second term of equation 12 gives a signal proportional to the model-plant error weighted by the control sensitivity function.

\section{Closed Loop Validation (Unstable Plants)}

When the model is from an unstable plant, the above schemes presented in figure 6 and figure 9 fail to provide a proper residual because slight model plant differences will lead to unbounded residual. Suitable structures to validate unstable models operating in closed loop are presented in figure 4 .

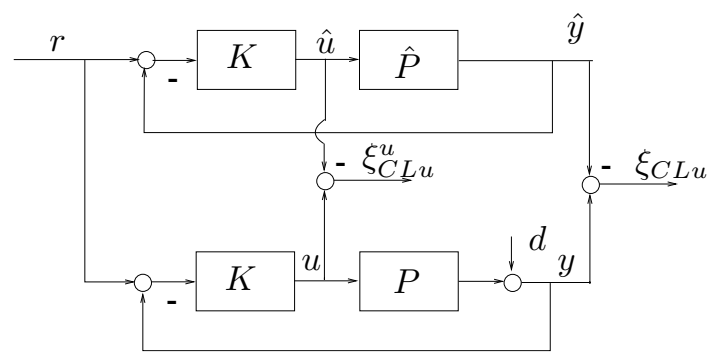

Fig. 4. Closed Loop Model Validation for Unstable Plants

The residual at the output $\xi_{C L u}$ (at the input $\xi_{C L u}^{u}$ ) of figure 4 are

$$
\begin{aligned}
& \xi_{C L u}=S d+K S \hat{S}(P-\hat{P}) r \\
& \xi_{C L u}^{u}=-K S d-K K S \hat{S}(P-\hat{P}) r
\end{aligned}
$$

Now, the residual $\xi_{C L u}\left(\xi_{C L u}^{u}\right)$ given by equation 8 (9) is always autocorrelated as it is filtered by the sensitivity function $S(K S)$ independently of the model-plant mismatch. The behaviour of the second term of equation 8 is similar to the ones explained above. Thus residuals generated under this structures are not suited to our approach. 
IV. Model Validation on Iterative Identification And Control Schemes

The benefits of the frequency dependent model validation for the iterative identification and control schemes hinge on the frequency domain information produced by the algorithm. It is possible to asses for what frequency range a new model should be identified (perhaps increasing the model order) and what frequency content should contain the input of the experiment. Moreover we have information over the frequency range for which the model is validated, thus it is possible to choose a proper controller bandwidth.

The benefits of the frequency dependent model validation approach over iterative control (see figure 5) are:

- Designing the input experiment for the next identification step. It is well known that the identified model quality hinges on the experiment designed to obtain the data. The experiment should contain high energy components on the frequency range where the model is being invalidated if informative data is pursued for a new identification in the following step.

- Detecting model undermodelling and/or choosing model order. A higher order model can be fitted over the frequency range where the current model is being invalidated. It can be done even inside the current iteration step without the need of performing a new experiment. In [12] a methodology to add poles and zeroes to an existing model can be found.

- Selecting controller bandwidth on the controller design step. Once a frequency range of the model has been validated, if no further improvement of the model is sought, the final controller designed should respect the allowable bandwidths of the model.

\section{EXAMPLES}

\section{A. Example 1}

The proposed validation procedure in the frequency domain is applied to a stable plant in open loop (see figure 6).

The real plant $G$ and the model $\hat{G}$ chosen to approximate it are:

$$
G=\frac{1}{(s+1)(s+10)}, \quad \hat{G}=\frac{1}{(s+1)}
$$

The Bode diagram comparing the real plant with the model is shown in figure 7. 


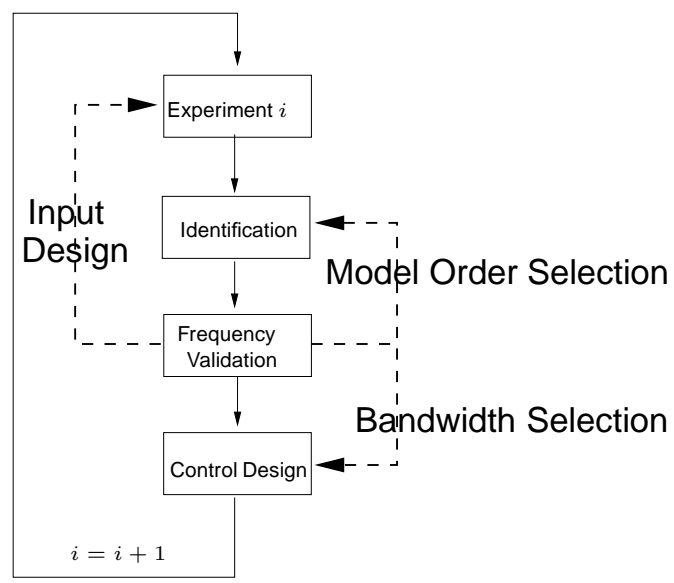

Fig. 5. Benefits of the frequency model validation approach on iterative identification and control schemes

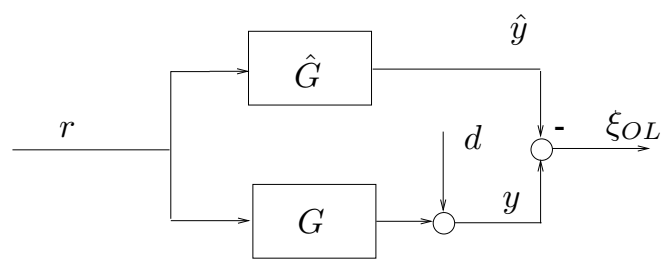

Fig. 6. Open Loop Model Validation

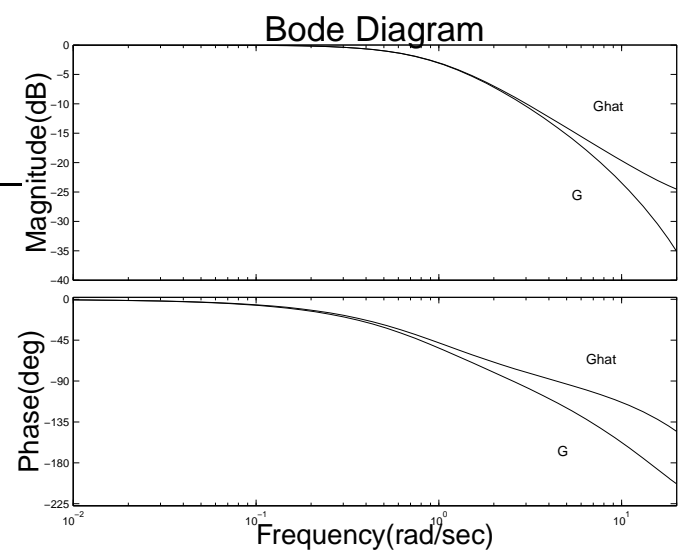

Fig. 7. Comparison of Bode diagrams

The experimental setup is as follows. The residuals generated by the open loop structure of 
figure 6 are given by

$$
\xi_{O L}=d+(G-\hat{G}) r
$$

Hence the residual $\xi_{O L}$ given by equation 11 is just the noise $d$ if the model and the plant are equal (i.e. $\hat{G}=G$ ). Hence the residual has the same stochastic properties than the noise.

The residuals corresponding to two different experiments with the same input are processed as described in section II-B. The perturbation $d$ is assumed to be white noise with $\sigma=1$. The reference input ris a train of sinusoids up to frequency $3 \mathrm{rad} / \mathrm{sec}$.

The validation procedure results can be seen in figure 8 . The model $\hat{G}$ shows no invalidation signs up to $1.4 \mathrm{rad} / \mathrm{sec}$. However for higher frequencies the hypothesis test fails to validate the model. As a conclusion we can state that, for the input applied, the model is correct for frequencies below to $1.4 \mathrm{rad} / \mathrm{sec}$. It is worth to mention that although between $1.7 \mathrm{rad} / \mathrm{sec}$ and $2.3 \mathrm{rad} / \mathrm{sec}$ there are no spikes out of the confidence limit, a deeper examination reveals that several consecutive spikes are abnormally high to belong a $\chi_{2}^{2}$ distribution. In order to detect this situations further probabilities should be checked (e.g. the probability that two consecutive points of a $\chi_{2}^{2}$ distribution be higher than some given value.)

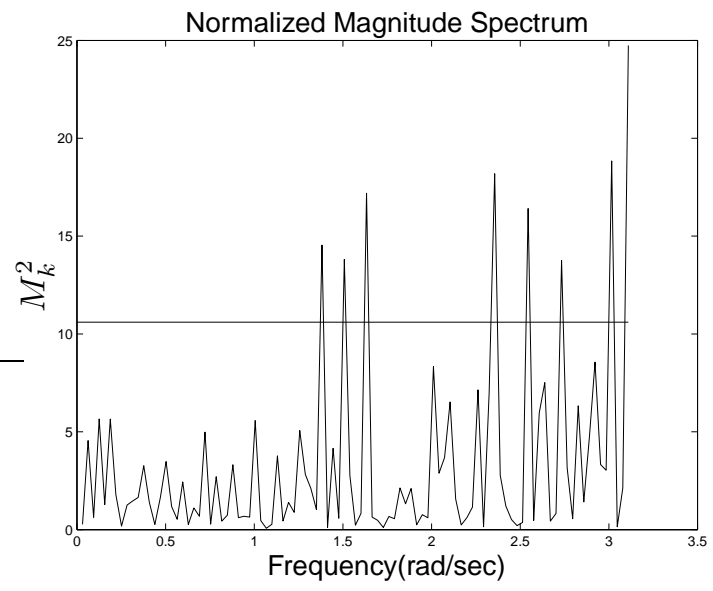

Fig. 8. Normalized Magnitude Spectrum and Confidence Limits

As a conclusion, the model $\hat{G}$ can be accepted as a good approximation of the plant $G$ up to frequency $1.4 \mathrm{rad} / \mathrm{sec}$. For higher frequencies the mismatch between model and plant is present up to the input bandwidth (i.e. $3 \mathrm{rad} / \mathrm{sec}$ ). It should be mention that this result is input dependent. 
However the results obtained up to now can serve as a guideline to design new input signals with suitable frequency contents for new identification steps (e.g. high energy around the frequencies were a significant error exists, that is between $1.4 \mathrm{rad} / \mathrm{sec}$ and $3 \mathrm{rad} / \mathrm{sec}$ ).

\section{B. Example 2}

The present example is the application of the proposed frequency domain model validation to an Iterative Control Design. As baseline we take the Iterative Control Design example presented in [7], page 126, where a stable plant with high-frequency resonant modes is controlled by successive plant identification (e.g. step response) and the subsequent controller design (e.g. model matching and cancellation controller). We apply to the successive models and controllers given in the example our frequency domain model validation procedure. Moreover we propose a customized structure in order to generate adequate residuals to claim for a control oriented model validation.

The proposed structure to generate the residuals is in closed loop, as shown in figure 9 .

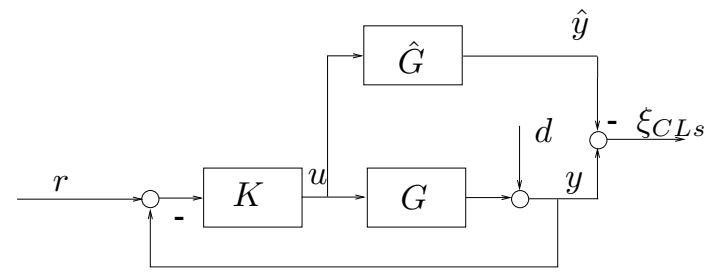

Fig. 9. Closed Loop Model Validation for Stable Plants

The residual is given by

$$
\xi_{C L s}=\frac{S}{\hat{S}} d+K S(G-\hat{G}) r
$$

The residual $\xi_{C L s}$ is the noise $d$ filtered by the fraction of the real Sensitivity function $S$ and the Sensitivity function of the model $\hat{S}$ plus a term that is the discrepancy of the plants weighted by the control sensitivity function. If the model and the plant are equal (i.e. $\hat{G}=G$ ) then the two sensitivity functions ( $S$ and $\hat{S}$ ) are equal so the first term of equation 12 yields the noise $d$. Moreover the second term, under the same perfect model-plant matching assumption, is zero. Hence the residual has the same properties of the noise $d$. However if there exist a discrepancy 
between the model and the plant then the division between $S$ and $\hat{S}$ is no longer the unity but a transfer function resulting in the noise $d$ filtered by $S / \hat{S}$. These facts makes the residual generated by this structure suitable for a whiteness test.

The experimental setup is as follows. First a model of the plant $\hat{G}$ is obtained by a step response identification. For this model successive controllers $K$ are designed by imposing more stringent reference models $M$. When the closed loop step response is unsatisfactory, a new model is identified and the controller design steps repeated. The measurement noise $d$ is white noise with $\sigma=10^{-2}$. The reference input $r$ is a train of sinusoids up to frequency $200 \mathrm{rad} / \mathrm{sec}$.

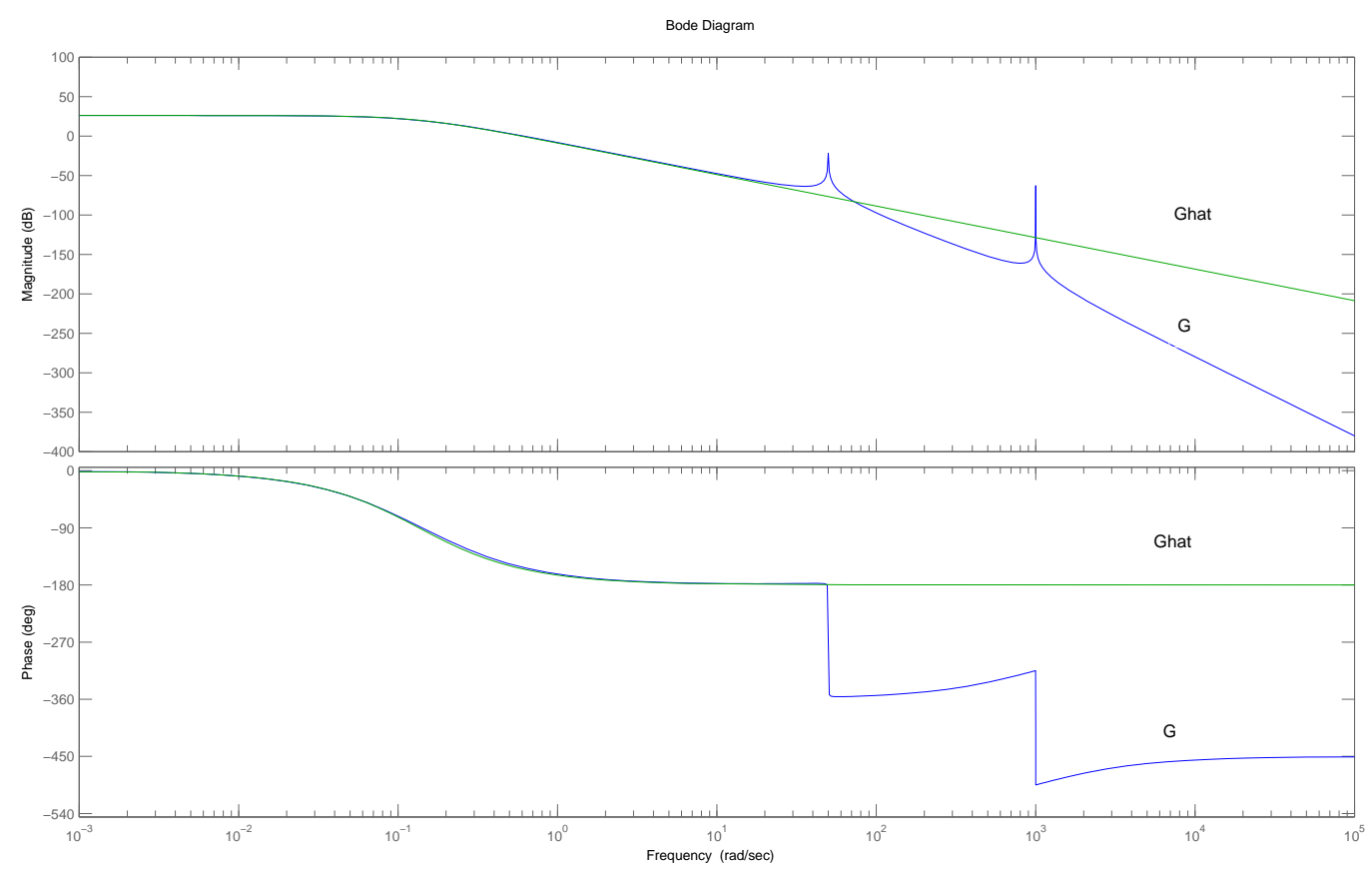

Fig. 10. Bode Plot

1) First Iteration: The first identified model and the model reference used to controller design are:

$$
\hat{G}_{0}=\frac{20}{(1+7.4 s)^{2}}, \quad M_{01}=\frac{0.5^{2}}{(s+0.5)^{2}}
$$

The bode plot of the real plant $G$ and the first model $\hat{G}_{0}$ is shown in figure 10 . The frequency domain validation is applied, given a positive validation result, as can be seen in the first plot 

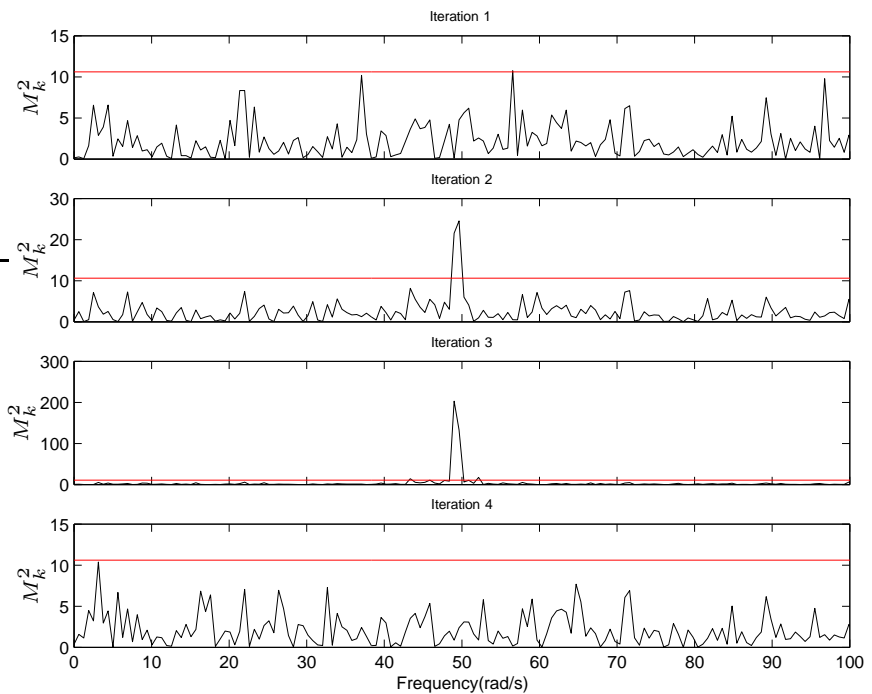

Fig. 11. Normalized Magnitude Spectrum and Confidence Limits

of figure 11 .

2) Second Iteration: Following the positive validation result of the first iteration the same model is kept as a valid one and the performance is pushed forward by a new, more stringent, reference model:

$$
\hat{G}_{0}=\frac{20}{(1+7.4 s)^{2}}, \quad M_{02}=\frac{3^{2}}{(s+3)^{2}}
$$

The validation test invalidate the model for frequencies around $50 \mathrm{rad} / \mathrm{sec}$ (see plot 2 of figure 11). This is due to the non modelled resonance peak as can be seen in the bode diagram of figure 10.

3) Third Iteration: In [7], the new identification step is taken after pushing even forward the desired reference model:

$$
\hat{G}_{0}=\frac{20}{(1+7.4 s)^{2}}, \quad M_{03}=\frac{5^{2}}{(s+5)^{2}}
$$

The invalidation of the model for frequencies around $50 \mathrm{rad} / \mathrm{sec}$ for this controller is evident (plot 3 of figure 11).

4) Fourth Iteration: In [7] a new model plant is identified due to the unacceptable closed loop behaviour for the controller designed with the reference model $M_{03}$. The new identified 
plant captures the first resonance peak of the plant.

$$
\begin{aligned}
\hat{G}_{1} & =\hat{G}_{0} \frac{0.01^{2}+50^{2}}{(s+0.01+50 i)(s+0.01-50 i)}, \\
M_{11} & =\frac{5^{4}}{(s+5)^{4}}
\end{aligned}
$$

The model validation result shows that now, the model is validated for all the frequency range covered by the input (plot 4 of figure 11).

Summarizing the example results, we have shown how the frequency dependent model validation scheme can be helpful to guide the identification step by aiming towards the interesting frequencies content that an identification experiment should excite. The procedure is helpful too to choose the appropriate controller bandwidth suitable for the actual model accuracy. Moreover it has been proven that the proposed methodology can be applied in iterative control design schemes and the validation can be control oriented.

\section{Example 3}

The objective of the present example is to compare our algorithm with the model validation proposed in the Windsurfer Approach [7]. In [9] the residual is calculated as shown in figure 4 and two complementary different validation methods are applied, a time domain method and a frequency domain method. The time domain is a classical cross-correlation test between the residual and the filtered input. In fact no whiteness test could be applied to the residual generated by the structure of figure 4 . The frequency domain method for model validation is based on comparing two power spectrum, the noise spectrum and the residual spectrum. By visual comparison of both spectrum it is possible to ascertain if the model is validated.

On the other hand our proposed algorithm calculates the residuals following structure in figure 9 and the validation procedure is the translation of a time domain whiteness test into a frequency domain one.

In [9] the following simulation example is proposed. The plant is a flexible link robot arm whose transfer function $G$ has poles at $s=-0.0996 \pm j 3.0017,-0.3339 \pm j 12.131$ and $-1.845 \pm$ $j 31.481$, zeros at $s=-13.162,-10.646 \pm j 12.27$ and $7.169 \pm j 11.54$ and $G(0)=0.5196$. The first model of the plant $G_{0}$ captures the first resonant frequency so $G_{0}$ has poles at $s=$ $-0.0903 \pm j 3.0027$, a zero at $s=-13.31$, and $G_{0}=0.5188$. The bode diagram of the plant and the model is shown in figure 12 . 


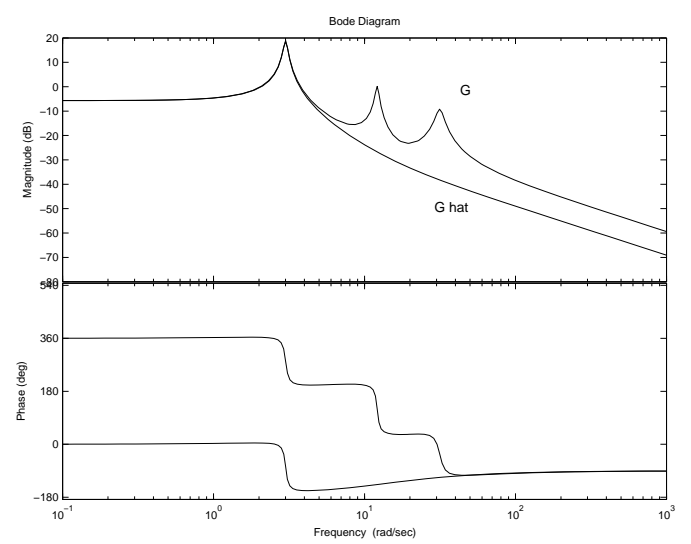

Fig. 12. Bode Plot

The controller is designed by the internal model control (IMC) method. The performance requirement is set by means of the closed loop bandwidth $\lambda$. When the parameter $\lambda$ is set equal to $1.5 \mathrm{rad} / \mathrm{sec}$ the validation method proposed in [9] gives the following results:

- The time domain cross-correlation method shows that $G_{0}$ is not a good model of $G$.

- The method of comparing the power spectra validates the model.

When the proposed algorithm is applied the validation result can be seen in figure 13. The model results invalidated around the frequency of $12 \mathrm{rad} / \mathrm{sec}$ (i.e. the second resonant frequency). The proposed approach has the following advantages:

- No time domain test is necessary. Classical time domain tests are not informative at all for iterative identification and control schemes.

- The frequency ranges for which the model results validated/invalidated are given in a precise form with a probability measure (i.e. hypothesis test).

\section{CONCLUSIONS}

A new approach for model validation on iterative identification and control schemes has been presented. The originality of the approach is that it validates the model in the frequency domain rather than in the time domain. The procedure of validating a model in the frequency domain has proven to be more informative for iterative control design schemes as it can serve as a guide for input experiment design (i.e. high energy content around frequencies where the model is 


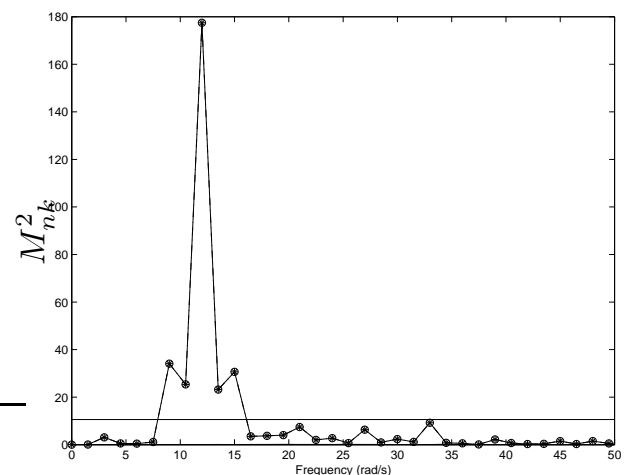

Fig. 13. Normalized Magnitude Spectrum and 99.5\% Confidence Limits

invalidated) and for controller design (i.e. limiting the controller bandwidth to those frequencies where an accurate model exists).

\section{APPENDIX}

\section{A. Proof of theorem 1}

The discrete Fourier transform of a discrete time signal $\xi_{k}$ is given by

$$
\xi_{k}=\frac{1}{N} \sum_{n=0}^{N-1} \xi(n) e^{-j \Omega_{0} k n}
$$

where $\Omega_{0}=\frac{2 \pi}{N}$ is the fundamental frequency. Decomposing the equation (17) into its real part and its imaginary part gives

$$
\begin{aligned}
\xi_{k} & =\frac{1}{N} \sum_{n=0}^{N-1} \xi(n) e^{-j \Omega_{0} k n} \\
& =\frac{1}{N} \sum_{n=0}^{N-1} \xi(n)\left(\cos \left(\Omega_{0} k n\right)-j \sin \left(\Omega_{0} k n\right)\right) \\
& =\frac{1}{N} \sum_{n=0}^{N-1} \xi(n) \cos \left(\Omega_{0} k n\right)-j \frac{1}{N} \sum_{n=0}^{N-1} \xi(n) \sin \left(\Omega_{0} k n\right) \\
& =R_{k}-j I_{k}
\end{aligned}
$$


where the second equality comes from Euler's identity. Hence the real and imaginary parts are linear combinations of normally distributed random variables

$$
\begin{aligned}
R_{k} & =\frac{1}{N} \sum_{n=0}^{N-1} \xi(n) \cos \left(\Omega_{0} k n\right) \\
I_{k} & =\frac{1}{N} \sum_{n=0}^{N-1} \xi(n) \sin \left(\Omega_{0} k n\right)
\end{aligned}
$$

As a result, it follows that $R_{k}$ and $I_{k}$ are also normally distributed random variables. The distribution parameters for the stochastic variable $R_{k}$ are calculated as follows (see, for example, [13], page 87 )

$$
\begin{aligned}
\mu_{R_{k}} & =\mu_{\xi} \frac{1}{N} \sum_{n=0}^{N-1} \cos \left(\Omega_{0} k n\right) \\
\sigma_{R_{k}}^{2} & =\sigma_{\xi_{n}}^{2} \frac{1}{N^{2}} \sum_{n=0}^{N-1} \cos ^{2}\left(\Omega_{0} k n\right)
\end{aligned}
$$

The same reasoning is applicable to the imaginary part $I_{k}$.

\section{B. Proof of theorem 2}

By definition the sum of " $r$ " independent squared random normal variables $N(0,1)$ has a $\chi^{2}$ distribution of $r$ degrees of freedom. Due to the normalization of $R_{k}$ and $I_{k}$, it follows that $\frac{R_{k}-\mu_{R_{k}}}{\sigma_{R_{k}}} \in N(0,1)$ and $\frac{I_{k}-\mu_{I_{k}}}{\sigma_{I_{k}}} \in N(0,1)$.

\section{REFERENCES}

[1] L. Ljung, System Identification. Theory for the User. Prentice-Hall, 1999.

[2] T. Soderstrom and P. Stoica, System Identification, M. Grimble, Ed. Prentice Hall International Series in Systems and Control Engineering, 1989.

[3] J. Chen and G. Gu, Control-Oriented System Identification. An $H_{\infty}$ Approach, S. Haykin, Ed. John Wiley \& Sons, Inc., 2000 .

[4] P. Balaguer and R. Vilanova, "Model validation on iterative identification and control schemes: A frequency domain approach," in 7th Portuguese Conference on Automatic Control, 2006.

[5] — - "Quality assessment of models for iterative/adaptive control," in 45th Conference on Decision and Control, 2006.

[6] L. Ljung, "System identification in a MIC perspective," Modelling, Identification and Control, vol. 15(3), pp. 153-159, 1994.

[7] P. Albertos and A. Sala, Eds., Iterative Identification and Control. Springer, 2002. 
[8] H. Hjalmarsson, "From experiment design to closed-loop control," Automatica, vol. 41, pp. 393-438, 2005.

[9] W. Lee, B. Anderson, I. Mareels, and R. Kosut, "On some key issues in the windsurfer approach to adaptive robust control," Automatica, vol. 31, pp. 1619-1636, 1995.

[10] R. Skelton, "Model error concepts in control design," International Journal of Control, vol. 49(5), pp. $1725-1753,1989$.

[11] M. Gevers, B. Codrons, and F. Bruyne, "Model validation in closed loop," in Proceedings of the American Control Conference, 1999.

[12] P. Balaguer and A. Ibeas, "Validation and improvement of models in the frequency domain," in IMACS Multiconference on Computational Engineering in Systems Applications, 2006.

[13] G. Box, W. Hunter, and J. Hunter, Statistics for Experimenters. An Introduction to Design, Data Analysis, and Model Building. John Wiley \& Sons, 1978. 